\title{
Helicobacter pylori infection and homocysteine levels in patients with cardiac syndrome $X$
}

We read the article "Lack of significant association between Helicob acter pylori infection and homocysteine levels in patients with cardiac syndrome X" written by Rasmi et al. [1] with a great interest. The authors pointed to the possible relation between H.pylori infection and homocysteine (Hcy) levels in cardiac syndrome X (CSX). They reported that plasma Hcy concentration in CSX patients is higher than the healthy subjects. There was no significant difference between Hcy in H.pylori + and H.pylori- individuals in CSX group and controls. Hcy levels in H.pylori+ individuals tend to be higher but it is not significant compared to H.pylori- individuals. Thank to the authors for their contribution of the present study successfully designed and presented. We believe that these findings will enlighten further studies about the relation between CSX and endothelial dysfunction.

CSX has 3 characteristic features; angina or angina like chest pain with exertion, ST segment depression that can be induced by treadmill exercise testing, or alternatively, a pathological thallium scan with normal coronary arteriography, and no spontaneous or inducible epicardial coronary artery spasm upon ergonovine or acetylcholine provocation [2].

$H$. pylori may also cause endothelial dysfunction directly by affecting the structure and function of the vascular endothelial cells via inflammation [3]. On the other hand, Hcy levels may be a risk factor, or an indicator of risk for the development of cardiovascular disease associated with endothelial dysfunction and inflammation [4].

In this point of view, the findings of the study should not be surprising. The study groups consisted of CSX and control subjects. According to the above determination of CSX, the authors did not use provocation test or hyperventilation maneuver for excluding spontaneous or inducible epicardial coronary artery spasm. Control subjects that may have in fact coronary artery disease were not appropriate because of not undergoing coronary angiography in this study. In addition, there may be differences in the parameters such as hypertension, hyperlipidemia, smoking and alcohol habits between the groups which could affect the results of the study to be mentioned to cause endothelial dysfunction.

Conflict of interest: none declared

\section{References}

1. Rasmi Y, Mehraban K, Sadreddini M et al. Lack of significant association between Helicobacter pylori infection and homocysteine levels in patients with cardiac syndrome X. Cardiol J, 2012; 19: 466-469.

2. Demirkol S, Balta S, Unlu M et al. Evaluation of the mean platelet volume in patients with cardiac syndrome X. Clinics (São Paulo, Brazil), 2012; 67: 1019-1022.

3. Rasmi Y, Raeisi S. Possible role of Helicobacter pylori infection via microvascular dysfunction in cardiac syndrome X. Cardiol J, 2009; 16: 585-587.

4. Agoston-Coldea L, Mocan T, Gatfosse M, Lupu S, Dumitrascu DL. Plasma homocysteine and the severity of heart failure in patients with previous myocardial infarction. Cardiol J, 2011; 18: 55-62. 